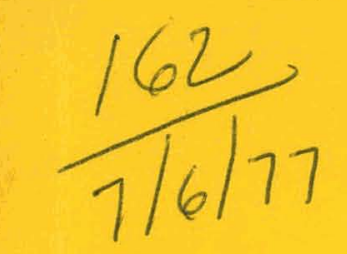

MLM-2403

MLM-2403

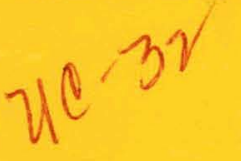

Stable Isotope Sales:

Mound Laboratory Customer and Shipment Summaries,

FY 1976 and FY 1976A

June 6, 1977

MOUND LABORATORY

Miamisburg, Ohio

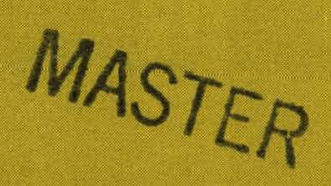

operated by

MONSANTO RESEARCH CORPORATION

a subsidiary of Monsanto Company

for the

UNITED STATES ENERGY RESEARCH

AND DEVELOPMENT ADMINISTRATION

U.S. Government Contract No. EY-76-C-04-0053 


\section{DISCLAIMER}

This report was prepared as an account of work sponsored by an agency of the United States Government. Neither the United States Government nor any agency Thereof, nor any of their employees, makes any warranty, express or implied, or assumes any legal liability or responsibility for the accuracy, completeness, or usefulness of any information, apparatus, product, or process disclosed, or represents that its use would not infringe privately owned rights. Reference herein to any specific commercial product, process, or service by trade name, trademark, manufacturer, or otherwise does not necessarily constitute or imply its endorsement, recommendation, or favoring by the United States Government or any agency thereof. The views and opinions of authors expressed herein do not necessarily state or reflect those of the United States Government or any agency thereof. 


\section{DISCLAIMER}

Portions of this document may be illegible in electronic image products. Images are produced from the best available original document. 


\section{NOTICE}

This report was prepared as an account of work sponsored by the United States Government. Neither the United States nor the United States Energy Research and Development Administration, nor any of their employees, nor any of their contractors, subcontractors, or their employees, makes any warranty, express or implied, or assumes any legal liability or responsibility for the accuracy, com-

pleteness or usefulness of any information, apparatus, product or process disclosed or represents that its use would not infringe privately owned rights. 


\title{
Stable Isotope Sales: Mound Laboratory Customer and Shipment Summaries, FY 1976 and FY 1976A
}

\author{
Compiled by Albin H. Ruwe, Jr.
}

Issued: June 6, 1977

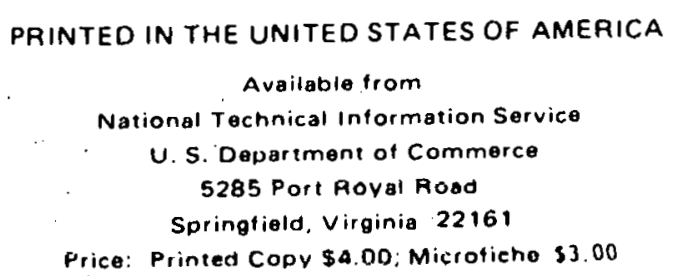

\section{MONSANTO RESEARCH CORPORATION}

A Subsidiary of Monsanto Company

\section{mound IABORETORY}

Miamisburg, Ohin

45342 .

operated for

\section{UNITED STATES ENERGY RESEARCH AND DEVELOPMENT ADMINISTRATION}

U. S. Government Contract No. EY-76-C-04-0053

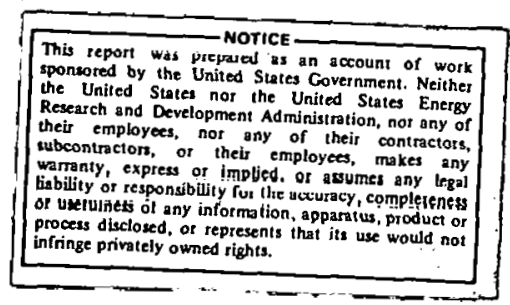




\section{Table of Contents}

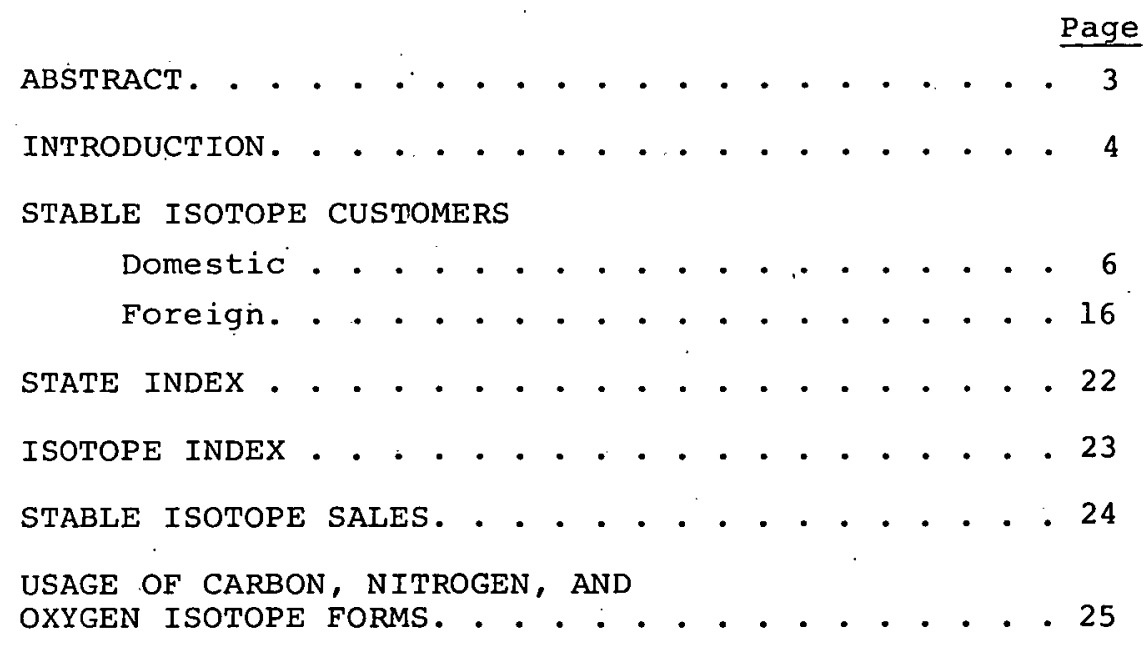




\title{
Abstract
}

\begin{abstract}
A listing is given of Mound Laboratory's sales of stable isotopes of noble gases, carbon, oxygen, nitrogen, chlorine, and sulfur for Fiscal Years 1976 and 1976A (the period July 1, 1975 through September 30,1976$)$. Purchasers are listed alphabetically and are divided into domestic and foreign groups. A cross-reference index by location is included for domestic customers. Cross-reference listings by isotope purchased are included for all customers.
\end{abstract}




\section{Introduction}

This report is the tenth in a series on Mound Laboratory customers and sales of stable isotopes and covers Fiscal Years 1976 and 1976A. Isotope separation by thermal diffusion was begun at Mound Laboratory in the late $1950^{\prime} \mathrm{s}$. Distribution of helium-3 directly to customers was begun in 1960; in 1964 distribution of neon, argon, krypton, and xenon stable isotopes and carbon-13 was started. The distribution of carbon-12 and oxygen16 was started in 1970. In 1972, 20\% carbon-13 was made available to supply customer needs. In 1973 distribution of nitrogen-14, nitrogen-15, oxygen-17, and oxygen-18 was started. The distribution of sulfur-34 was started in 1975. Chlorine distribution commenced in 1976.

All the noble gas isotopes were isotopically enriched by thermal diffusion in cascaded columns. Carbon-13 and carbon-12 were enriched by carbon monoxide distillation. Carbon-13 enriched methane was enriched from 908 to $97 \%$ and 998 by a thermal diffusion column cascade. Carbon isotopes were produced by both Mound Laboratory and Los Alamos Scientific Laboratory (LASL) and distributed by Mound Laboratory. The isotopes of oxygen and nitrogen were enriched by nitric oxide distillation at Lns Alamos Scientific Laboratory for distribution by Mound Laboratory. Sulfur and chlorine isotopes were enriched by liquid thermal diffusion at Mound Laboratory.

This summary was compiled to present the sales of stable isotopes of the noble gases, carbon, oxygen, nitrogen, chlorine, and sulfur, which were produced for the U. S. Energy Research and Development Administration at Mound Laboratory and Los Alamos Scientific Laboratory for the Fiscal Years 1976 and 1976A (July 1, 1975 through Sept. 30, 1976).
The customer tabulations are divided into the following sections:

- An alphabetical list of customers that includes, as far as practical, contacts at the buyer's plant. This section is further subdivided into domestic and foreign listings.

- An alphabetical list of states with cross references to customer numbers.

- An alphabetical list of isotopes with cross references to customer numbers.

The sales tabulations are divided into two parts. Part one shows total sales and furnishes the isotope, total number of shipments, total quantity, and the total value (including cylinder, packaging, and handling) of isotope shipments in Fiscal Years 1976 and 1976A. Part two furnishes similar but more detailed information for carbon, nitrogen, oxygen, and sulfur isotopes.

The available concentrations of the enriched isotopes have varied over a wide range. For example, xenon-124 has been enriched to 648 , whereas helium- 3 has been enriched to 99.99968 .

The stable isotopes were distributed at enrichments noted in Table 1 .

The stable isotopes of carbon, nitrogen, oxygen, chlorine, and sulfur were distributed in the forms shown in Table 2 .

Helium-3 continued to be the major stable isotope in terms of volume and dollar sales of the numerous stable isotopes produced at Mound Laboratory. Information on enrichments, procurement, and prices may be obtained by contacting:

\author{
Monsanto Research Corporation \\ Stable Isotope Sales \\ Mound Laboratory \\ Miamisburg, Ohio 45342 \\ Commercial telephone: \\ 513-866-7444, Ext. 3502, 7337 \\ Federal Telecom: \\ $774-3502,7337$
}

Commercial teletype:

TWX No. 810-47342974 
Table 1

Enrichment, Mole 8

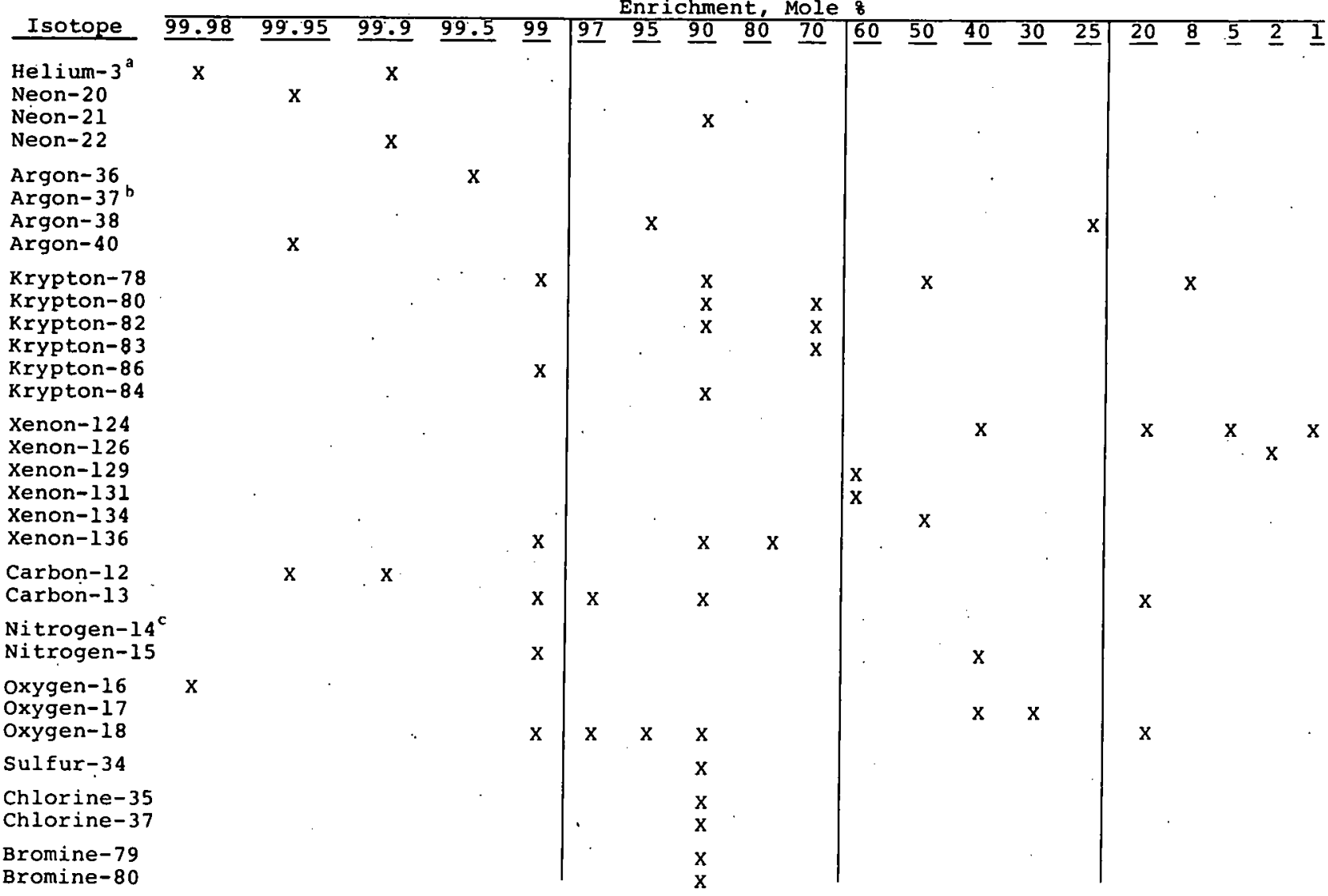

${ }^{a}$ Helium-3 also distributed at 99.99958 enrichment.

Argon-37 distributed at 70-fold enrichment.

${ }^{\mathrm{c}} \mathrm{Nitrogen-14}$ distributed at 99.998 enrichment.

Table 2

\begin{tabular}{|c|c|c|c|c|c|c|c|c|c|c|c|c|}
\hline \multirow[b]{2}{*}{ Compound } & \multicolumn{12}{|c|}{ Isotope } \\
\hline & $\mathrm{C}-12$ & $c-13$ & $\underline{N-14}$ & $\underline{\mathbf{N}-15}$ & $0-16$ & $\underline{n-17}$ & $\underline{Q-18}$ & $s=34$ & $B r-79$ & $\underline{B r-80}$ & $\mathrm{Cl}-35$ & $\mathrm{Cl-37}$ \\
\hline Ammonia & & & & $\mathrm{x}$ & & & & & & & & \\
\hline Ammonium nitrate & & & $\mathrm{x}$ & & . & & & & & & & \\
\hline Ammonium sulfate-solid & & & & $\mathrm{x}$ & & & ' & & & & & \\
\hline $\begin{array}{l}\text { Ammonium sulfate-solution } \\
\text { Barium carbonate }\end{array}$ & & & $x$ & & & & & & & & & \\
\hline $\begin{array}{l}\text { Barium carbonate } \\
\text { Carbon dioxide }\end{array}$ & $\begin{array}{l}x \\
x\end{array}$ & $\begin{array}{l}x \\
x\end{array}$ & & & & & & & & & & \\
\hline Carbon monoxide & $\hat{x}$ & $\hat{x}$ & & & & & 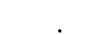 & & & & & \\
\hline $\begin{array}{l}\text { Carbon disulfide } \\
\text { Elemental }\end{array}$ & & & & & & & & $x$ & & & & \\
\hline Carbon & $\mathrm{x}$ & $\mathrm{x}$ & & & & - & & & & & & \\
\hline Chlorine (gas) & & & & & & & & & & & $\mathrm{x}$ & $x$ \\
\hline Nitrogen (gas) & & & & $\mathrm{x}$ & & & & & & & & \\
\hline $\begin{array}{l}\text { Oxygen (gas) } \\
\text { Sulfur }\end{array}$ & & . & & & $\mathrm{x}$ & & $x$ & & & & & \\
\hline $\begin{array}{l}\text { Sulfur } \\
\text { Hydrogen sulfide }\end{array}$ & & & & & & & & $\mathrm{x}$ & & & & \\
\hline $\begin{array}{l}\text { Hydrogen sulfide } \\
\text { Metháne }\end{array}$ & & $\mathrm{x}$ & & & & & & $\mathrm{x}$ & & & & \\
\hline $\begin{array}{l}\text { Methane } \\
\text { Nitric acid }\end{array}$ & & & & $x$ & & & & & & & & \\
\hline Potassium nitrate & & & & $\mathrm{x}$ & & & & & & & & \\
\hline Water & & & . & & $\mathrm{x}$ & $\mathrm{x}$ & $\mathrm{x}$ & & & & & 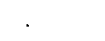 \\
\hline Sulfur dioxide & & & & & & & & $\mathrm{x}$ & & & & . \\
\hline 3ulfurio acid? & & & & & & & & $\mathrm{x}$ & & & & \\
\hline Ethyl bromide & & & & & & & & & $\mathrm{x}$ & $\mathrm{x}$ & & \\
\hline Chlorobenzene & & & & & & & & & & & $x$ & $\pi$ \\
\hline Hydrogen chloride & & & & & & & - & & & & $\mathrm{x}$ & $\mathrm{x}$ \\
\hline Sodium chloride & & & & & & & & & & & $\mathrm{X}$ & $\mathrm{x}$ \\
\hline
\end{tabular}




\title{
FY-1976 and FY-1976A
}

\section{Stable Isotope Customers}

\author{
Where no isotope is listed \\ helium-3 was the only isotope purchased.
}

\section{Domestic}

1. Aerojet Nuclear Co.

550 Second Street

Idaho Falls, ID 83401

J. K. Saari

$\mathrm{C}-13\left(\mathrm{BaCO}_{3}\right), \mathrm{Kr}-78, \mathrm{Kr}-86$

2. Aerospace Corporation

$200 \mathrm{~N}$. Douglas street

El Segundo, CA 90245

D. James

$0-18 \quad\left(\mathrm{O}_{2}\right)$

3. Airco Industrial Gases

River \& Union Landing Roads

Riverton, NJ 08077

Mr. Gussman

4. Andros, Inc.

2332 Fourth street

Berkeley, CA 94710

C. Martin

$\mathrm{C}-13 \quad\left(\mathrm{CO}_{2}\right)$

5. Argonne National Laboratory

9700 South Cass Avenue

Argonne, IL 60439

R. Damm, J. G. Daunt, J. Frogge

J. Blaise, E. Kwirant,

C. Rutkowski

$\mathrm{He}-3, \mathrm{C}-13\left(\mathrm{C}, \mathrm{CO}_{2}\right)$

$\mathrm{Kr}-80, \mathrm{Kr}-82, \mathrm{Kr}-83, \mathrm{Kr}-84, \mathrm{Kr}-86$

$\mathrm{O}-18\left(\mathrm{H}_{2} \mathrm{O}, \mathrm{O}_{2}\right)$

6. Arizona State University

Zoology Department

Tempe, AZ 85281

N. F. Hadley

$0-18\left(\mathrm{H}_{2} \mathrm{O}\right)$

7. Battelle Memorial Institute

505 King Avenue

Columbus, OH 43201

R. N. Eddy

R. Winchell

O-16 $\left(\mathrm{O}_{2}\right), \mathrm{O}-18\left(\mathrm{H}_{2} \mathrm{O}\right)$

8. Battelle Northwest

P. O. Box 999

Richland, WA 99352

S. Rogers

$\mathrm{Xe}-124$
9. Bell Telephone Labs, Inc.

600 Mountain Avenue

Murray Hill, NJ 07974

R. F. Dartnell

C. J. Mogab

$\mathrm{He}-3, \mathrm{~N}-\mathrm{I} 5\left(\mathrm{~N}_{2}\right)$

10. Bio-Rad Laboratories

2400 Wright Avenue

Richmond, CA 94084

B. Llewellyn Gabel $\mathrm{N}-15 \quad\left[\begin{array}{lll}\left(\mathrm{NH}_{4}\right)_{2} & \mathrm{SO}_{4}\end{array}\right]$

11. Boston University

Physics Department

111 Cummington street

Boston, $M \lambda 02215$

Mr. Zimmerman

12. Brandeis University

Lab. Supp. \& Services

Waltham, MA 02154

H. I. McCarthy

13. Brookhaven National Laboratory Isotopes and Special Materials Building $\mathrm{T}-89$

Upton, LI, NY 11973

P. Van Wormer

$\mathrm{He}-3, \mathrm{Ar}-36, \mathrm{Kr}-78$

14. Brooklyn college

Dept. of Chemistry

Bedford Ave. \& Avenue $\mathrm{H}$

Brooklyn, NY 11210

T. Ishida, H. Wieck

$\mathrm{C}-13\left(\mathrm{CO}_{2}\right)$

15. CW Radiation, Inc. 111 Ortega Avenue Mountain View, CA 94040 D. B. Perkins

16. C. W. Radiation, Inc. 140 Kifer Court Sunnyvale, CA 94086 K. Sample 
17. California, University of

2000 Carleton Street

Berkeley, CA 94720

Mr. Brilliant

18. California, University of

Lawrence Berkeley Laboratory

End of Hearst Avenue

Berkeley, CA 94720

J. T. Haley, J. T. Beales

$\mathrm{He}-3, \mathrm{Ar}-36, \mathrm{Ne}-22, \mathrm{O}-18 \quad\left(\mathrm{H}_{2} \mathrm{O}\right)$

$\mathrm{Kr}-84, \mathrm{Kr}-86$, $\mathrm{Xe}-136$

$\mathrm{C}-12\left(\mathrm{BaCO}_{3}\right), \mathrm{C}-13\left(\mathrm{BaCO}_{3}\right)$

19. California, University of

Davis, CA 95616

J. T. Huber

Dept. of Animal Science

$\mathrm{N}-15\left(\mathrm{NH}_{4}\right)_{2} \mathrm{SO}_{4}$

C. Chan

Dept. of Agronomy and Range Science

$\mathrm{N}-15\left(\mathrm{NH}_{4}\right)_{2} \mathrm{SO}_{4}$

D. J. Duthie

Chemistry Dept.

$\mathrm{N}-15\left(\mathrm{NH}_{3}\right)$

S. Winebaum

Environmental Health \& Safety

$\mathrm{N}-15$ ( $\mathrm{KNO}_{3}$ )

D. Duthie

Physics Dept.

$\mathrm{He}-3$

P. Stout

Soils and Plant Nutrition

$\mathrm{N}-15\left(\mathrm{KNO}_{3}, \mathrm{~N}_{2},\left(\mathrm{NH}_{4}\right)_{2} \mathrm{SO}_{4}\right)$;

$\mathrm{N}-14\left(\mathrm{NH}_{4}\right)_{2} \mathrm{SO}_{4}$

20. California, University of

School of Physical Science

Irvine, CA 92664

W. Hehre

L. Travis

$0-18 \quad\left(\mathrm{O}_{2}\right)$

21. California, University of

Physies Department

3175 Miramar Road

La Jolla, CA 92037

W. Stephens

22. California, University of

Lawrence Livermore Laboratory

7000 East Avenue

Livermore, CA 94550

C. B. Sanford

$\mathrm{Ne}-22, \mathrm{Kr}-82, \mathrm{Kr}-83, \mathrm{Xe}-136$, $0-18\left(\mathrm{H}_{2} \mathrm{O}\right)$

23. California, University of

Laboratory of Nuclear Medicine

900 Veteran Avenue

Los Angeles, CA 90024

E. B. Jones

$\mathrm{He}-3, \mathrm{Kr}-82, \mathrm{O}-18 \quad\left(\mathrm{H}_{2} \mathrm{O}\right)$

$\mathrm{N}-15^{2}\left[\left(\mathrm{NII}_{4}\right)_{2} \mathrm{SO}_{4}, \mathrm{KNO}_{3}\right]$
24. California, University, at L.A.

741 Circle Drive S

Los Angeles, CA 90024

E. B. Jones

C. J. Neagle, O. L. Chapman

$\mathrm{He}-3, \mathrm{C}-\mathrm{I} 3\left(\mathrm{BaCO}_{3}\right)$

25. California, University of

Los Alamos Scientific Laboratory

Los Alamos, NM 87545

R. J. Van Gemert, E. F. Wartmann,

L. Guthrie, R. Froberg

$\mathrm{He}-3, \mathrm{Ne}-20, \mathrm{O}-18\left(\mathrm{O}_{2}\right)$

$\mathrm{C}-13,\left(\mathrm{C}, \mathrm{CH}_{4}\right)$

26. University of California

3401 Watkins Drive

Riverside, $\mathrm{CA} 92507$

$\mathrm{N}-15\left(\mathrm{NH}_{4}\right)_{2} \mathrm{SO}_{4}$

27. California, Institute of Technology 391 S. Holliston

Pasadena, CA 91106

R. L. Mooney, Z. E. Switkowski

R. W. Kavanagh, R. Blackwood

$\mathrm{C}-12(\mathrm{C}), \mathrm{S}-34(\mathrm{~S})$

28. California State University, L.A.

5151 State University Drive

Los Angeles, CA 90032

$\mathrm{H}$. Winkler

$\mathrm{Ne}-21$

29. Southern California, University of Dept. of Physics

Los Angeles, CA 90007

H. Bozler

30. Case Western Reserve University

2232 Circle Drive

Cleveland, OH 44106

L. O. Major, Jr.

D. G. Pohlman, L. O. Major, Jr.

G. Mateescu

$\mathrm{He}-3,0-18\left(\mathrm{O}_{2}\right)$

$\mathrm{N}-15\left(\mathrm{~N}_{2}, \mathrm{KNO}_{3}\right)$

31. Catholic University of America, N.E. Department of Physics

Washington, DC 20064

M. T. Lunnón

$\mathrm{Ne}-22$

32. University of Chicago

933 E. 56 th. Street

Chicago, IL

9. Tcjero

33. Chicago, University of

Franklin McLean Mem. Res. Ins.

5827 Drexel Blvd.

Chicago, IL 60637

P. V. Harper

$\mathrm{He}-3, \mathrm{Kr}-80$ 
34. Clarkson College

Dept. of Physics

Potsdam, NY

A. Czanderná

$\mathrm{He}-3$, $\mathrm{Ne}-20, \mathrm{O}-18\left(\mathrm{O}_{2}\right)$

35. Coherent Radiation

3210 Porter Drive

Palo Alto, CA 94304

Carol Green, M. Winnburg

36. Colorado, University of Nuclear Physics Laboratory Boulder, CO 80309

J. L. Homan, C. D. Zafirates

$\mathrm{He}-3, \mathrm{Kr}-78, \mathrm{Kr}-80, \mathrm{Kr}-82$

$\mathrm{Kr}-83, \mathrm{Kr}-84, \mathrm{Xe}-132$

37. Colorado State University

Department of Chemistry

Fort Collins, $\mathrm{CO} 80523$

C. E. Maciel, A. S. Greenberg

$\mathrm{He}-3, \mathrm{C}-13\left(\mathrm{BaCO}_{3}\right)$

38. The Connecticut Agriculture

Experiment Station

Dept. of Soil and.Water

123 Huntington street

New Haven, CT 06504

$\mathrm{N}-14\left[\left(\mathrm{NH}_{4}\right)_{2} \mathrm{SO}_{4}\right]$

39. Cornell University

69 Clark Hall

Ithaca, NY 14853

B. Nathan, E. Smith, R. Buhrman

40. Cutler-Hammer, Inc.

A.I.L. Division

Walt Whitman Road

Melville, NY

A. Stain

$\mathrm{C}-13\left(\mathrm{CO}_{2}\right)$

41. Cyrogenic Rare Gas Labs., Inc.

730 South 13 th Street

Newark, NJ 07103

W. Dietz

42. Drexel University

34 th \& Ludlow streets

Philadelphi, PA 19104

J. C. Scanlon

$\mathrm{C}-13\left(\mathrm{CO}_{2}\right), \mathrm{Kr}-86$

43. Duke University

Department of Physics

Durham, NC 27706

H. W. Newsom, H. Meyer, R. Byrd $\mathrm{He}-3, \mathrm{~N}-15\left(\mathrm{~N}_{2}\right)$

44. Duke Medical Center

Durham, NC 27710

J. Miller

$\mathrm{C}-13(\mathrm{CO})$
45. EG\&G, Inc.

\& U. S. ERDA

130 Robin Hill Rd.

Goleta, CA 93017

D. F. McCue

46. E. I. DuPont Co.

Experimental Station

Wilmington, $\mathrm{DE} 19898$

R. Brown, W. E. Yound

$\mathrm{C}-13\left(\mathrm{CO}, \mathrm{CO}_{2}, \mathrm{BaCO}_{3}\right)$

47. E. I. dupont de Nemours \& Co. Savannah River Plant

Aiken, SC 29801

Rodman, M. L. Hyder

J. T. Buchner

$\mathrm{C}-13(\mathrm{CO}), 0-16\left(\mathrm{O}_{2}\right)$

48. East Texas State University

Chemistry Dept.

Commerce, TX 75428 $0-18(\mathrm{H}, \mathrm{O})$

49. Eco-Control, Inc.

Key Isotopes

56 Rogers Street

Cambridge, MA 02142

$\mathrm{N}-15\left(\mathrm{NH}_{3}\right), \mathrm{C}-13(\mathrm{C})$

50. Albert Einstein College of Medicine 1300 Morris Park Avenue

Rronx, N.Y. 10461

M. Fabry

$\mathrm{C}-13\left(\mathrm{CO}_{2}\right)$

51. El Don Company

USA

2876 Butternut street

Ann Arbor, MI 48104

D. E. Gillespie

52. Energy Research \& Development Adm.

\&U. S. Bureau of Mines

Cochrans Mill Road

Bruceton, PA 15213

$\mathrm{C}-12(\mathrm{C}), \mathrm{C}-13(\mathrm{C}), \mathrm{S}-34\left(\mathrm{H}_{2} \mathrm{~S}\right)$

53. Dept. of Environmental Quality

Division of water Quality

1234 W. Morrison

Portland, OR 97205

v. Dunnette

$\mathrm{N}-15\left[\left(\mathrm{NH}_{4}\right)_{2} \mathrm{SO}_{4}\right]$

54. Exxon Research and Enqineering

$1600 \mathrm{E}$. Linden Avenue

Linden, NJ 07036

D. Rapp

$\mathrm{O}-18\left(\mathrm{H}_{2} \mathrm{O}, \mathrm{O}_{2}\right)$ 
55. Florida, University of

Gainesville, FL 32610

D. Silverman, College of Medicine

$0-18\left(\mathrm{H}_{2} \mathrm{O}\right)$

D. N. Silverman, Pharmacology

$\mathrm{C}-13\left(\mathrm{C}, \mathrm{BaCO}_{3}\right), \mathrm{O}-18\left(\mathrm{H}_{2} \mathrm{O}\right)$

E. D. Adams, Physics Dept.

$\mathrm{He}-3$

J. Messer, E. Holtzclaw, J. Carr $\mathrm{He}-3, \mathrm{~N}-15\left(\mathrm{NH}_{4}\right)_{2} \mathrm{SO}_{4}$

56. Fusion Energy Corporation

3684 U. S. Route 1

Princeton, NJ 08540

J. Nering

57. GTE Sylvania, Inc.

Johnston Street

Seneca Falls, NY 13148

$\mathrm{Ne}-20$

58. General Electric Company

Neutron Devices Dept.

Byran Dairy Road

St. Petersburg, FL 33733

H. I. Kolinski

59. General Electric

50 Fordham Road

Wilmington, MA 01887

Bob Maitnes

O-16 $\left(\mathrm{H}_{2} \mathrm{O}\right)$

60. University of Georgia

Safety Services Dept.

Athens, GA 30602

L. Muse, M. J. Cormier

Physics Dept., L. Arnold

$\mathrm{He}-3, \mathrm{O}-18\left(\mathrm{H}_{2} \mathrm{O}\right)$

61. Grumman Aerospace Corp.

Bethpage, NY 11714

F. Kuehne

62. Hanscom AFB

MA 01731

ESD Bldg. 1614 LGSBR

B. Duffy

$\mathrm{Xe}-1.36$

63. Harshaw Chemical Company

6801 Cochran Road

Solon, OH 44139

J. Zamiska

64. Harvard University

Dept. of Chemistry

12 oxford street

Cambridge, MA 02138

$\mathrm{J}$. Knowles

$\mathrm{O}-18\left(\mathrm{H}_{2} \mathrm{O}\right)$

65. Hewlett Packard Company

Sallta Clara Divisinn

5301 Stevens Clark Blvd.

Santa Clara, CA 95050

M. Hopson

$\mathrm{He}-3, \mathrm{Ne}-20$
66. Honeywell, Inc.

2600 Ridgeway

Minneapolis, MN 54413

T. I. Wallerus

$\mathrm{He}-3$, Ne-2l, $\mathrm{Ne}-22$

67. Honeywell, Inc.

1433 Stinson Blvd.

Minneapolis, MN 55413

$T$. Wallerus

$\mathrm{He}-3$, Ne-20

68. Hope College

Physics Dept.

Holland, MI 49423

B. Hichwa

69. John Hopkins University

62.2 North: Washington street

Baltimore, MD 21205

J. J. Kaufman, D. Correl1

$0-18\left(\mathrm{O}_{2}\right), \mathrm{N}-15\left[\mathrm{~N}_{2}, \mathrm{INH}_{3}\right.$,

$\left.\left(\mathrm{NH}_{4}\right)_{2} \mathrm{SO}_{4}, \mathrm{KNO}_{3}\right]$

70. Hughes Aircraft Co.

Centinela \& Teale Streets

Culver City, CA 90230

N. Kilstein

$\mathrm{C}-13\left(\mathrm{CO}_{2}\right)$

71. Hughes Aircraft Company

Electron Dynamics Division

3100 West Lomita Blvd.

Torrance, CA 90509

B. McKaig, L. Freedman

$\mathrm{He}-3$, Ne-20, $\mathrm{Ne}-22$

72. Hughes Laser Products

6155 El Camino Real

Carlsbad, CA 92008

H. Zimmerman

$\mathrm{He}-3, \mathrm{Ne}-22$

73. IBM Corporation

6300 Diagonal Highway

Boulder, CO 80302

E. Arnold, C. B. Little

$\mathrm{He}-3, \mathrm{Ne}-22$

74. IBM Research Lab

5600 Cattle Road

San Jose, CA 95193

T. D. Sappington

75. IBM-T. J. Watson Research Center

Kitchawan Route-Route 134

Yorktown, NY 10598

R. J. Arculeo

S. E. Burks

$\mathrm{He}-3, \mathrm{Ne}-20$

76. Illinois, University of

1609 South Oak Street

Champaign, IL 61820

M. L. Eapoznik

$\mathrm{He}-3, \mathrm{C}-13\left(\mathrm{CO}, \mathrm{BaCO}_{3}\right), \mathrm{O}-18\left(\mathrm{H}_{2} \mathrm{O}\right)$ 
77. Illinois, University of

Urbana, IL 61801

D. M. Ginsberg, McKinley Hosp.

A. Akerman, Nuclear Eng.

$\mathrm{N}-15 \quad\left(\mathrm{KNO}_{3}\right)$

78. Indiana, University of

1025 East Ilth Street

Bloomington, IN 47401

P. L. Smith

$\mathrm{O}-18\left(\mathrm{H}_{2} \mathrm{O}, \mathrm{O}_{2}\right) \mathrm{C}-13\left(\mathrm{CO}_{2}\right), \mathrm{N}-15\left(\mathrm{~N}_{2}\right)$

79. Indiana, Univer of Pennsylvania

Weyandt Hall

Indiana, PA 15701

80. Institute of International Education

809 United Nations Plaza

New York, NY 10017

N. Brady

$\mathrm{N}-\mathrm{I5}\left(\mathrm{NH}_{4}\right)_{2} \mathrm{SO}_{4}$

81. Jodon Engineering Assoc., Inc.

145 Enterprise Drive

Ann Arbor, MI 48103

J. W. Gillespie

$\mathrm{Ne}-22$

82. Kansas State University

Department of Agronomy

Manhattan, KS 66506

R. V. Olson

$\mathrm{N}-15\left[\left(\mathrm{NH}_{4}\right)_{2} \mathrm{SO}_{4}, \mathrm{~N}_{2}\right]$

$\mathrm{C}-13\left(\mathrm{CH}_{4}\right)$

83. C. F. Kettering Research Laboratory

150 East South College Street

Yellow Springs, OH 45387

W. E. Newton

$0-18 \quad\left(\mathrm{H}_{2} \mathrm{O}\right)$

84. Koch Isotopes, Inc.

72 Rogers street

Cambridge, MA 02142

M. Zyberg, M. Wyman

$\mathrm{N}-15\left[\mathrm{HNO}_{3},\left(\mathrm{NH}_{4}\right)_{2} \mathrm{SO}_{4}, \mathrm{~N}_{2}\right]$

$\mathrm{C}-13\left(\mathrm{CO}_{2}, \mathrm{CO}, \mathrm{C}, \mathrm{CH}_{4}\right)$

$\mathrm{C}-12\left(\mathrm{CO}_{2}, \mathrm{CO}\right), \mathrm{O}-18\left(\mathrm{H}_{2} \mathrm{O}\right)$

85. LND, Incorporated

3230 Lawson Blvd.

Oceanside, NY 11572

W. Muzzey

86. Landseas Corp.

138-0.1 Springfiled Blvd.

Jamaica, NY 11413

M. Freindlich

87. Lasermetrics, Inc.

ll1 Galway Place

Teaneck, NJ

R. Goldstein, A. Idelson

$\mathrm{O}-17\left(\mathrm{O}_{2}\right), \mathrm{O}-18\left(\mathrm{H}_{2} \mathrm{O}\right)$

$\mathrm{N}-15\left[\left(\mathrm{NH}_{4}\right)_{2} \mathrm{SO}_{4}\right]$

$\mathrm{C}-13\left(\mathrm{BaCO}_{3}, \mathrm{C}\right)$
88. Liconix

1400 Stierlin Road

Mountain View, CA 94043

K. Funch, Feltman

89. Litton Systems, Inc.

Guidance \& Control Systems Div.

5500 Canago Avenue

Woodland Hills, CA 91364

D. Howarth, S. Norrell,

J. Schroeder

$\mathrm{He}-3, \mathrm{Ne}-20, \mathrm{Ne}-22$

$\mathrm{Kr}-83, \mathrm{C}-13\left(\mathrm{CO}_{2}\right)$

90. Lockheed Missiles \& Space Co., Inc.

3251 Hanover Street

Palo Alto, CA 94304

91. Maryland, University of

College Park, MD 20742

Mr. Glover

92. University of Massachusetts

Nuclear Physics

Amherst, MA

G. A. Peterson

93. Massachusetts Inst. of Technology Cambridge, MA 02139

D. Reed

$0-18\left(\mathrm{O}_{2}\right)$

94. Massachusetts Inst. of Technoloyy

Lincoln Laboratory

244 Wood Street

Lexington, MA 02173

C. Freed, A. Bernstein

$\mathrm{C}-13\left(\mathrm{CO}_{2}, \mathrm{C}\right)$

95. Mathesoil Gas

8800 Utica Avenue

Cucamonga, CA 91730

$\mathrm{C}-13(\mathrm{CO})$

96. Matheson Gas Products

932 Paterson Plank Road

E. Rutherford, NJ 07073

Pat Taylor

B. Steinhardt

$\mathrm{He}-3, \mathrm{Ne}-20, \mathrm{Ne}-22, \mathrm{Kr}-82$

97. Metrologic Instruments, Inc.

143 Harding Avenue

Bellmawr, NJ 08030

C. H. Knowles

98. University of Michigan

3072 Phoenix Memorial Laboratory

2301 Banisteel Blvd.

Ann Arbor, MI 48109

C. W. Ricker, W. Miller

99. Michigan State University

$1330 \mathrm{~S}$. Harrison Road

East Lansing, MI 48824

D. R. Merritt, J. R. Snook

$\mathrm{He}-3, \mathrm{Ar}-36$

$\mathrm{C}-13\left(\mathrm{C}, \mathrm{CO}_{2}\right)$ 
100. Midwest Research Institute

425 Volker Blvd.

Kansas City, MO 64110

W. P. Duncan

$\mathrm{C}-13(\mathrm{CO})$

101. Minnesota, University of

Williams Lab. of Nuclear Physics

830 E. River Flats

Minneapolis, MN 55455

G. F. Schissel

J. H. Broadhurst

$\mathrm{He}-3, \mathrm{O}-18\left(\mathrm{H}_{2} \mathrm{O}, \mathrm{O}_{2}\right), \mathrm{N}-15\left(\mathrm{~N}_{2}\right)$

102. Minnesota, University of

Physics Depart

Chemistry Dept.

Minneapolis, MN 55455

Dr. P. Gassman

W. Weyhmaan

$\mathrm{He}-3, \mathrm{C}-13\left(\mathrm{CO}_{2}\right)$

103. University of Minnesota

Dept. of Soil Science

st. Paul, MN 55108

G. E. Ham

$\mathrm{N}-15\left[\left(\mathrm{NH}_{4}\right)_{2} \mathrm{SO}_{4}\right]$

104. University of Missouri

Dept. of Chemistry

St. Louis, MO 63121

K. W. Barnett

$\mathrm{C}-13(\mathrm{CO})$

105. Monsanto Company

$800 \mathrm{~N}$. Lintherg Blvd.

St. Louis, MO 63166

J. Schaefer,

H. Frazier, M. Sadorf

$\mathrm{N}-15\left[\left(\mathrm{NH}_{4}\right)_{2} \mathrm{SO}_{4}\right], \mathrm{C}-13(\mathrm{CO})$

$\mathrm{S}-34\left(\mathrm{CS}_{2}\right)$

106. Monsanto Research Corporation

Mound Laboratory

Miamisburg, OH 45342

D. Pardieck

107. National Aeronautics \& Space Admin.

Langley Research Center

Hampton, VA 23665

C. R. Lane

L. Cannon

108. National Aeronautics \& Space Admin.

Tewis Research Center

21000 Brookpark Road

Cleveland, OH 44135

J. W. Blue

$\mathrm{Xe}-131$

109. National Bureaul of Standards

Route 270 \& Quince Orchard Road

Gaithersburg, MD 20234

K. violer

$\mathrm{Xe}-129$
110. National Institute of Health Radioisotope Laboratory

Bldg 21

Bethesda, MD 20014

H. Kon

$\mathrm{N}-15\left(\mathrm{~N}_{2}\right)$

111. National Institute of Neurological and Communicative Disorders \& Stroke Laboratory of Neuropharmacology 9000 Rockville Pike Bethesda, MD 20014 M. J. Perlow, R. Kartzinel O-18 $\left(\mathrm{O}_{2}\right), \mathrm{N}-15\left(\mathrm{~N}_{2}\right)$

112. Naval Research Laboratory

Washington, D. C. 20375

Ms. Bell, W. Whitney

E. Altman

$\mathrm{He}-3, \mathrm{C}-13\left(\mathrm{CO}_{2}, \mathrm{CO}\right)$

113. University of Nevada, Las Vegas

4505 Maryland Parkway

Las Vegas, NV 89154

L. D. Spignt

114. New England Nuclear

64 Industrial Way

Wilmington, MA 01887

D. Dumas

C $-13(\mathrm{C})$

115. New York at Albany, State U. of Dept. of Physics

1400 Washington Ave.

Albany, NY .12222

J. Greene, C. P. Scholes

$0-17\left(\mathrm{O}_{2}\right), \mathrm{Cl}-37(\mathrm{NaCL})$

116. New York at Buffalo, St. U. of

Physics Dept.

250 Winspear Avenue

Buffalo, NY 14214

F. Gasparini

117. New York at Stony Brook, st. U. of Stony Brook, NY 11796

E. Schultz, Physics

$\mathrm{C}-13(\mathrm{C}), 0-18\left(\mathrm{O}_{2}\right)$

118. New York University

Physics Department

4 Washington Place

New York, NY 10003

5. J.'Williamson

119. North Carolina State University Soil Science Dept.

Raleigh, NC.

D. W. Israel

$\mathrm{N}-15\left(\mathrm{~N}_{2}\right)$ 
120. North Dakota State University

Dept. of Soils

Walster 104

Fargo, ND 58102

C. Smith

$\mathrm{N}-15\left(\mathrm{KNO}_{3}\right)$

121. North Texas State University

2204 West Prairie

P. O. Box 13766

Denton, TX 76203

J. R. Marshall

$\mathrm{Mr}$. Welch

$\mathrm{C}-13\left(\mathrm{CO}_{2}\right)$

122. Northwestern University

2145 Sheridar Road

Evanston, IL 60201

I. M. Klotz, Chemistry

J. McDowell, Physics

123. Notre Dame University

Dept. of Physics

Notxe Dame, IN 46556

$\mathrm{J}$. Duncan

$0-18\left(\mathrm{O}_{2}\right)$

124. Ohio State Univ. Research Found.

1314 Kinnear Road

Columbus, OH 43212

R. Warwick

$\mathrm{He}-3, \mathrm{C}-13\left(\mathrm{BaCO}_{3}\right)$

125. Ohio State University

Physics Department

Smith Laboratory

2009 Millikan Road

Columbus, $\mathrm{OH} 43210$

D. F. Stobbs, M. Yaqub

$\mathrm{He}-3, \mathrm{C}-13\left(\mathrm{BaCO}_{3}\right)$

126. Oklahoma State University

Department of Chemistry

Stillwater, OK 74074

J. Best

$\mathrm{O}-18\left(\mathrm{O}_{2}\right)$

127. Oregon, University of

Physics Dept.

Eugene, OR 97403

R. J. Higgins

P. E. Gallagher, Chem. Dept.

H. J. Evans

$\mathrm{Ne}-13, \mathrm{C}-13\left(\mathrm{BaCO}_{3}\right), \mathrm{N}-15\left[\left(\mathrm{NH}_{4}\right)_{2} \mathrm{SO}_{4}\right]$

128. Ortec, Inc.

100 Midway Lane

Oak Ridge, TN 37830

V. Martin

129. Owens-Illinois, Inc.

Technical Center

1700 North Westwood Avenue

Toledo, OH 43607

B. Bobbitt

$\mathrm{He}-3, \mathrm{Ne}-20, \mathrm{Ne}-22$
130. Pathfinder Laboratories

11542 Fort Mims Drive

S.t. Louis, MO 63141

M. E. Johns

$\mathrm{C}-13\left(\mathrm{CO}_{2}, \mathrm{C}\right)$

131. Pennsylvania, University of

Dept. of Physics

David Rittenhous Lab.

209 South 33 rd Street

Philadelphia, PA 19174

S: C. Headley

$$
\mathrm{O}-18\left(\mathrm{O}_{2}\right)
$$

132. Pennsylvania State University Department of Biochemistry

219 Shields Bldg

8 Althouse Laboratory

University Park, PA 16802

J. J. Villafranca

C. R. Hartzell

$\mathrm{C}-13(\mathrm{CO} 2)$

133. Perkin-Elmer

50 Danbury Road

Wilton, CT 06897

P. Panzarino

$\mathrm{C}-13\left(\mathrm{CO}_{2}\right)$

134. Pfizer Minerals, Pigments \& Metal Division

640 North 13th

Easton, PA

P. Wood

$\mathrm{C}-13\left(\mathrm{CH}_{4}\right)$

135.: Pittsburgh, University of

Terrace and Desoto street

Pittsburgh, PA 15261

S. Batchen

$\mathrm{He}-3, \mathrm{C}-13\left(\mathrm{BaCO}_{3}\right)$

136. Princeton Univ.

Jadwin Hall

Washington Road

Princeton, NT 08540

Mr. Roman

137. PROCHEM

47 Meadowbrook Place

Maplewood, NJ 07040

J. W. Kilby

C-13 $\left(\mathrm{CH}_{4}\right), 0-18\left(\mathrm{O}_{2}\right)$

138. Purdue University

West Lafayette, IN 47907

P. H. Keesom

139. Radio Corporation of America New Holland Pike

Lancaster, PA 17604

D. A. Kirchner

140. Radio Corporation of America David Sarnoff Research Center Princeton, NJ 08450

T. Pruss 
141. Raytheon Company

Research Division

28 Seyon street

Waltham, MA 02154

J. Swift

$\mathrm{He}-3, \mathrm{Ne}-20, \mathrm{Ne}-22$

142. Raytheon Company

Equipment Division

Equipment Dev. Labs

Boston Post Road

Wayland, MA 01778

W. D. Cronin

$\mathrm{He}-3, \mathrm{Ne}-20, \mathrm{Ne}-22$

143. Reuter-Stokes

18530 South Miles Parkway

Cleveland, $\mathrm{OH} 44128$

N. Callahan, J. Skarupa

K. McCormick

144. Rice University

Bonner Nuclear Lab.

Physics Dept.

Houston, TX 77001

D. May

145. Rochester, University of NSRL-Tandem

271 East River Road

. Rochester, NY 14623

R. L. Burns

$\mathrm{He}-3, \mathrm{O}-18\left(\mathrm{H}_{2} \mathrm{O}\right)$

146. Rockwell International

nutonetirs Division

3195 E. LaPalma

Anaheim, CA 92803

F. Vescial

$\mathrm{He}-3, \mathrm{Ne}-20, \mathrm{Ne}-22$

147. Rockwell International Corp.

Atomics International Division 8.900 Desoto Avenue

Canoga Park, CA 91304

H. Farrar

148. S.H.E. Corporation

1166 I Sorrento Valley Road

San Diego, CA 92121

E. Hirschkoff

149. Sandia Laboratories

Kirtland Air Force Base (East)

Albuquerque, NM 87115

J. P. Callahan

150. San Francisco State University

1600 Hollaway Avenue

San Francisco, CA 94132

B. Cowan

$\mathrm{N}-15,\left[\left(\mathrm{NH}_{4}\right)_{2} \mathrm{SO}_{4}\right]$
151. Savannah River Ecology Laboratory, Special Supplies

Savannah River Plant

Aiken, SC 29801

J.: B. Woods

$\mathrm{O}-18\left(\mathrm{H}_{2} \mathrm{O}\right)$

152. The Singer Co.

1225 McBride Ave

Little Falls, NJ 07424

J. Simpson, C. Olfers

$\mathrm{He}-3$, Ne-20, $\mathrm{Ne}-22$

153. Smithsonian Institution Astrophysical Observatory 185 Alewife Brook Parkway Cambridge, MA 02138

154. Spectra-Physics, Inc. 1250 West Middlefield Road Mountain View, CA 94042

N. Claus, H. Nazarewicz $\mathrm{He}-3, \mathrm{Ne}-2 \mathrm{O}$

155. Stanford Research Institute Bldg. 324, 4 th street Menlo Park, CA 94025 R. Kable $\mathrm{O}-18\left(\mathrm{O}_{2}\right)$

156. Stanford University Physics Department Stanford, CA 94305 S. H. Hill

157. Stevens Institute of Technology Cryogenics Center Hobokan, NJ. 07030

158. Stohler Isotope Chemicals

Los Alamos Division

Trinity Square Plaza

Los Alamos, NM 87544 $\mathrm{N}-15\left(\mathrm{NH}_{3}\right), \mathrm{C}-13\left(\mathrm{CO}, \mathrm{CO}_{2}\right)$

159. Stohler Isotope Chemicals, Inc. 49 Jones Road

Waltham, MA 02154

J. Barry

$\mathrm{N}-15\left[\left(\mathrm{NH}_{4}\right)_{2} \mathrm{SO}_{4}, \mathrm{~N}_{2}\right]$

$\mathrm{O}-18\left(\mathrm{H}_{2} \mathrm{O}\right), \mathrm{C}-12\left(\mathrm{CO}_{2}\right)$

$\mathrm{C}-13\left(\mathrm{BaCO}_{3}, \mathrm{CO}\right), \mathrm{O}-17\left(\mathrm{H}_{2} \mathrm{O}, \mathrm{O}_{2}\right)$

160. Synchemex

2860 Detroit Avenue

Cleveland, OH 44113

W. J. Ferguson

$\mathrm{N}-15\left(\mathrm{NH}_{4}\right)_{2} \mathrm{SO}_{4}$

161. Temple University

Barton Hall

13 th \& Norris Streets

Philadelphia, PA 19122

Dr. J. r.row 
162. Tennessee, Univ. of

Physics \& Astronomy Dept.

Knoxville, TN 37916

N. Gailar, C. King

$\mathrm{C}-13\left(\mathrm{CH}_{4}\right)$

163. Tennessee Valley Authority

Div. of Agriculture Development

Div. of Chemical Development

Muscle Shoals, AL 35660

G. Wiezcurak

$\mathrm{N}-14\left[\left(\mathrm{NH}_{4}\right)_{2} \mathrm{SO}_{4}\right]$

164. Tennessee Valley Authority

National Fertilizer Dev. Center

Muscle Shoals, AL 35660

G. A. Wieczorek

$\mathrm{N}-14\left[\left(\mathrm{NH}_{4}\right)_{2} \mathrm{SO}_{4}\right]$

165. Texas at Austin, Univ. of

Physics Department

Robert Lee Moore Hall

Austin, TX 78722

C. Walton

166. Texas at Dallas, Univ. of

Box 688, 2601 North Floyd Rd.

Richardson, TX 75080 .

K. Duerksen

$\mathrm{Kr}-86, \mathrm{Xe}-136$

167. Texas A\&M University

Chemistry Department

College Station, TX 77843

J. R. Kolb

J. Beckham, Physics Dept.

$\mathrm{He}-3, \mathrm{C}-13(\mathrm{CO})$

168. Texas A\&M University

Cyclotron Institute

College Station, TX 77843

J. A. Treat

Ar -36

169. Texas Instruments, Inc.

13510 North Central Expressway

Dallas, TX 75231

B. Morton, H. Woodard

$\mathrm{He}-3, \mathrm{O}-18\left(\mathrm{H}_{2} \mathrm{O}\right)$

170. Texas Nuclear Corporation

9101 Highwày 183

Austin, TX 78766

C. D. Wallin.

171. Tufts University

Physics Dept.

Medford, MA 02155

H. H. Sample

172. Tulane University

1301 Audubon Street

New Orleans, LA 70118

D. J. Dareksbourg

$\mathrm{C}-13(\mathrm{CO}), \mathrm{O}-18\left(\mathrm{H}_{2} \mathrm{O}\right)$
173. Union Carbide Corp.

P. O. Box 237 Industrial Highway Keasbey, NJ 08832

E. Juskis

$0-18\left(\mathrm{H}_{2} \mathrm{O}\right)$

174. Union Carbide Corporation

Nuclear Division

Oak Ridge National Laboratory

Oak Ridge, TN 37830

M. F. Phillips, B. J. Copeland

W. R. Osborn, Mr. Spooner,

Mr. King

$\mathrm{He}-3, \mathrm{Ne}-22, \mathrm{~N}-15\left(\mathrm{~N}_{2}\right)$

$\mathrm{C}-13\left(\mathrm{CO}_{2}, \mathrm{C}\right), \mathrm{O}-16\left(\mathrm{O}_{2}\right), 0-18\left(\mathrm{O}_{2}\right)$

175. U. S. Dept. of Agriculture, ARS

ARC-West

Beltsville, MD 20705

$\mathrm{J}$. Legg

$\mathrm{N}-15\left[\left(\mathrm{NH}_{4}\right)_{2} \mathrm{SO}_{4}\right]$

176. U. S. Geological Survey

Federal Center

Denver, CO 80225

$\mathrm{J}$. Yaggie

$\mathrm{O}-16\left(\mathrm{H}_{2} \mathrm{O}\right), \mathrm{C}-12(\mathrm{C})$

177. U. S. Services, Inc.

19 Ox Bow Lane

Summit, NJ

$\mathrm{J}$. Kilby

$\mathrm{Ne}-22$

178. University of Utah

311 A Skaggs Hall

Salt Lake City, UT 84112

$\mathrm{J}$ : A. McCloskey

A. Frank

$\mathrm{O}-18\left(\mathrm{H}_{2} \mathrm{O}\right), \mathrm{N}-15\left(\mathrm{NH}_{3}\right)$

179. Washington, University of

Chemistry Department

Nuclear Physics Laboratory

Seattle, WA 981.95

R. Burk, B. Meyer

$\mathrm{O}-18\left(\mathrm{H}_{2} \mathrm{O}\right), \mathrm{C}-13\left(\mathrm{CH}_{4}\right)$

$\mathrm{S}-34(\mathrm{~S}), \mathrm{N}-15\left(\mathrm{~N}_{2}, \mathrm{NH}_{3}\right)$

180. Washington University

Cyclotron Laboratory

6540 Millbrook

St. Louis, MO 63130

J. T. Hood

181. Washington University Medical School Dept. of Psychiatry

499 S. Euclid

st. Louis, MO 63110

P. Hipps

$\mathrm{O}-18\left(\mathrm{H}_{2} \mathrm{O}\right)$ 
182. Wesleyan University

Physics Dept.

Middletown, CT

R. Rollefson

183. Westinghouse Hanford Co.

P. O. Box 1970

Richland, WA 99352

G. C. Peterson

$\mathrm{Kr}-78$

184. Wisconsin, University of

750 University Avenue

Madison, WI 53706

P. Beighley, Physics

H. A. Schimming, Chemistry

R. J. Nickles, Medical Physics

C. R. Hutchinson, Pharmacy

$\mathrm{He}-3, \mathrm{O}-18\left(\mathrm{H}_{2} \mathrm{O}, \mathrm{O}_{2}\right)$

$\mathrm{C}-13\left(\mathrm{BaCO}_{3}\right)$

185. Wright-Patterson Air Force Base

Air Force Avionics Laboratory

Dayton, OH 45433

$\mathrm{He}-3, \mathrm{Ne}-20, \mathrm{Ne}-22$

186. Xerox Electro-Optical Systems

250 N. Halstead Street

Pasadena, CA 91107

V. F. Johnson

$\mathrm{He}-3, \mathrm{Ne}-20$
187. Yale University

College Plaza

47 College St., Suite 207

New Haven, CT 06510

D. Sadler

$\mathrm{C}-13\left(\mathrm{BaCO}_{3}\right)$

$\mathrm{O}-1.8 \quad\left(\mathrm{O}_{2}\right)$

188. Yale University

260 Whitney Avenue

New Haven, CT 06520

F. Curtis

$\mathrm{N}-15\left(\mathrm{~N}_{2}\right), \mathrm{C}-13\left(\mathrm{CO}_{2}\right)$

189. Daniel F. Young, Inc. 176-20 147th Avenue Jamaica, NY 11434

A. Breslin

$\mathrm{Ne}-20, \mathrm{C}-13\left(\mathrm{BaCO}_{3}\right)$

190. Zyberg, Michael

123 Woodland Avenue Westwood, NJ 07675 $\mathrm{C}-13\left(\mathrm{CO}_{2}\right), \mathrm{N}-15\left[\left(\mathrm{NH}_{4}\right)_{2} \mathrm{SO}_{4}\right]$ 


\title{
FY-1976 and FY-1976A
}

\section{Stable Isotope Customers}

\author{
Where no isotope is listed \\ helium- 3 was the only isotope purchased.
}

\section{Foreign}

\section{AUSTRALIA}

191. Adelaide, Univ. of

Adelaide 5000

Dept. of Physical \& Inorganic Chemistry

South Australia

P. J. Dunlap

192. CSIRO National Measurements Lab.

Remington Centre

183 Liverpool street

Sydney, NSW 2000, Australia

F. J. Lehany

$\mathrm{He}-3, \mathrm{Ne}-22$

193. Sydney University Grounds

The Receiving Store

CSIRO National Measurements Lab.

City Road, Chippendale

Sydney, Australia

$\mathrm{Ne}-20$

194. State Stores Board

50 Margaret st.

Brisbane, Queensland, 4000

Australia

Mr. Houghton

$\mathrm{N}-14\left[\left(\mathrm{NH}_{4}\right)_{2} \mathrm{SO}_{4}\right]$

\section{AUSTRIA}

195. International Research Establishment 1238 Vienna Anton Kriegerg 155

Vienna, Austria

Mr. Gazda

$\mathrm{Kr}-82$

\section{BELGIUM}

196. CEN/SCK

Boeretang 200

P. B. 2400 Mol

Belgium

A. Falla
197. Institut Voor Kern-en Stralingsfysika Celestijnan Laan $200 \mathrm{D}$ 3030 Heverlee Belgium

P. Mariens,

198. Universite de Louvain Institut de Physique Chemin du Cyclotron, 2 1348 Louvain-La-Neuve, Belgium

J. Remy, A. Hatert

$\mathrm{He}-3, \mathrm{O}-18\left(\mathrm{H}_{2} \mathrm{O}\right)$

\section{BRAZIL}

199. Universidade Est adual de Campinas, Campinas Instituto de Fisiça Cao Paulo, Brazil

C. Rettori

\section{CANADA}

200. Alberta, University of

Nuclear Research Centre Eiliuutun, Alberta, Canada J. Davidson, R. Fairweather, Mr. Cameron, Nuclear Physics $\mathrm{He}-3, \mathrm{Kr}-84, \mathrm{Kr}-86, \mathrm{C}-13(\mathrm{CO})$

201. Atomic Energy of Canada, Ltd. Chalk River Nuolcar Laburatorles Chalk River, Ontario, Canada J. P. S. Pringle, J. Muff, D. Millar, A. H. Allwright $\mathrm{He}-3, \mathrm{Ar}-36, \mathrm{~N}-15\left(\mathrm{~N}_{2}\right)$ $\mathrm{S}-34\left(\mathrm{H}_{2} \mathrm{~S}\right), \mathrm{O}-\mathrm{I} 8\left(\mathrm{H}_{2} \mathrm{O}\right)$

202. Atomic Energy of Canada, Ltd. Commercial Products Tunney's Pasture ottawa, Ontario, Canada W. J. Craig, W. Whyte $\mathrm{C}-13$ (C) 


\section{CANADA}

203. British Columbia, University of Physics Department

Vancouver 8

British Columbia V6T 1W5, Canada E. MCLintock

204. Manitoba, University of

Physics - Cyclotron

Winnipeg, Manitoba, Canada

A. N. Sourkes

$\mathrm{Ne}-20$

205. McGill University

Foster Radiation Lab.

P. O. Box 6070

Montreal, P.Q. H3C 3Gl

Canada

S. K. Mark

$\mathrm{Kr}-78, \mathrm{Xe}-124$

$\mathrm{O}-18\left(\mathrm{H}_{2} \mathrm{O}\right)$

206. McMaster University

General Sciences

Hamilton, Ontario, Canada L8S $4 \mathrm{Kl}$ P. Ikossi

$$
s-34(S)
$$

207. Merck Frosst Laboratories

16711 Route Transcanadienne

Pte, Claire

Kirkland, Quebec, Canada.

M. Cool, R. R. Menendez,

J. Ethier

$\mathrm{C}-13\left(\mathrm{CO}_{2}, \mathrm{BaCO}_{3}, \mathrm{CO}\right)$

O-18 $\left(\mathrm{H}_{2} \mathrm{O}\right), \mathrm{S}-34(\mathrm{~S})$

208. Universite de Montreal

Laboratoire de Physique Nucleare

2900 Boul. Ed. Montpetit cH: 103

Montreal, P.Q. Canada

C. Brassard

209. University of Montreal

Nuclear Physics Laboratory

2905 Marie Guyard

Montreal, Canada

C. Brassard

210. National Research Council

Physics Division

Montreal Road

ottawa, Ontario KlA Osl

Canada

Mr. Hoolahand

$\mathrm{C}-13(\mathrm{C}), \mathrm{N}-15\left(\mathrm{~N}_{2}\right)$

211. National Research Council

Chemistry Division

100 Susses Drive

Ottawa, Ontario KlA OR6

$0-18\left(\mathrm{H}_{2} \mathrm{O}\right)$
212. University of Toronto

Dept. of Physics

McLennan Physical Labs

Huron/Russell Sts. Toronto, Ontario

Canada

A. D. May

213. University of Quebec

INRS-Energie

1650 Montee Ste Julie

C. P. 1020

Varennes, Quebec, Canada

A. Jacob

214. Queens University

Physics Dept.

Kingston, Ontario, Canada $S-34(S)$

\section{DENMARK}

215. University of Aarhus

Institute of Physics

DK-8000 Aarhus C

Denmark

H. L. Nielsen, T. Anderson

$\mathrm{He}-3, \mathrm{~N}-15\left(\mathrm{~N}_{2}\right)$

216. Niels Bohr Institutet

Tandem Accelerator Laboratoriet

Riso pr. 4000

Roskilde, Denmark

$J$. Westergaard

$\mathrm{C}-13\left(\mathrm{CH}_{4}\right)$

\section{ENGLAND}

217. Birmingham, Univ. of

Department of Physics

P. O. Box 363

Birmingham, B15 2'L Erit Eland Ar-38

218. Bristol, Univ. of

Medical School

University Walk, Bristol BS 8 LTD.

United Kingdom

D. R. Trentham

$\mathrm{O}-18\left(\mathrm{H}_{2} \mathrm{O}\right)$

219.: Dyson Perrins Laboratory

South Parks Road

Oxford OX1 3QY England

G. Lowe

$\mathrm{O}-17\left(\mathrm{H}_{2} \mathrm{O}\right), \mathrm{O}-18\left(\mathrm{H}_{2} \mathrm{O}\right)$

220. University of Glasgow

Dept. of Natural Philosophy

University Avenue, Glasgow G12 $8 \mathrm{QQ}$

Scotland

R. Singhal

$\mathrm{Kr}-84, \mathrm{Kr}-86, \mathrm{~S}-34\left(\mathrm{H}_{2} \mathrm{~S}\right)$ 
ENGLAND

221. Liverpool, University of

Dept. of Physics

oxford street

Liverpool L69. 3BX England

L. I. Green

Ar-38, Xe-129

222. Manchester, University of

Physics Dept.

Schuster Lab

Manchester, England

H. E. Hall, W. R. Phillips

$\mathrm{He}-3, \mathrm{Ne}-22$

223. Ministry of Agriculture Fisheries and Food

Block C Government Buildings

Brooklands Avenue

Cambridge CB2 20R England

T. H. Caldwell

$\mathrm{N}-14 \quad\left[\left(\mathrm{NH}_{4}\right)_{2} \mathrm{SO}_{4}\right]$

224. Oxford, University of

Dept. of Nuclear Physics

Keple Road

Oxford, OXI $3 \mathrm{RH}$, England

$\mathrm{K}$. W. Allen

Dr. Sinclair

$\mathrm{C}-12\left(\mathrm{CO}, \mathrm{CH}_{4}\right), \mathrm{O}-18\left(\mathrm{H}_{2} \mathrm{O}\right)$

225. Radiochemical Centre

Amershaul, Bucks, England

A. M. McCallum

$\mathrm{Xe}-124$

226. Scientifica \& Cook Electronics Limited

78 Bollo Bridye Road

noton

London W3 8AU, England

P. D. Cook

$\mathrm{Ne}-22$

227. U K Treasury \& Supply Delegation 25 Broadway

New York, NY 10004

Mr. Ashman, M. Chambers,

N. Joseph

$\mathrm{N}-15:\left[\left(\mathrm{NH}_{4}\right)_{2} \mathrm{SO}_{4}, \mathrm{KNO}_{3 .}\right]$

\section{FRANCE}

228. C.E.A. Dept. Recherche et Analyse Bureau des Isotopes Stables

B.P. No. 2-91190 Gif-Sur-Yvette \% Bansad-Aeroport Charles de Gaulle Roissy-France MME Pauly

$\mathrm{Kr}-86, \mathrm{Xe}-136, \mathrm{C}-13\left(\mathrm{CO}, \mathrm{CO}_{2}, \mathrm{BaCO}_{3}\right)$ $\mathrm{S}-34$
229. Commissariat A L'Energie

Atomique

Dept. Des Radioelements

B P No. 2-91190 Gif-Sur-Yvette

Orly, France

Mr. Bussman, F. Botter, C. Pauly

Dept. of Research \& Analysis

$\mathrm{C}-13(\mathrm{C})$

230. Institut de Physique Nucleaire de Lyon

Universite claude Bernard

69621 Villeurbanne

France

N. Chevaier

$\mathrm{Ne}-22$

231. Institut Max Von Lave

B.P. 156

38 Grenoble Cedex

France

$\mathrm{J}$. Julien

Ar-36

\section{WEST GERMANY}

232. Amersham Buchler GMBH \& Co. KG 3 Harxbuettelgr strasse 3301 Wenden via Braunschweig West Germany $\mathrm{Kr}-80$

233. Gesellschaft fur Kernfurschung mbH-I sotopenstelle

75 Karlsruhe

Weverstrasse 5, West Ccrmary

A. Wolf

$\mathrm{He}-3, \mathrm{Kr}-82, \mathrm{~N}-15\left(\mathrm{~N}_{2}\right)$

234. Institut fur Experimental Physik

III. Ruhr-Universitat Bochum

Universitatsstr, 150, Geb. NB

463 Bochum/Germany

Postfach 2148

H. Buttlar

Ar -36

235. Institut f. Kernphysik

Von-Esinarch st. $10 a$

44 Munster, Germany

C. Rolfs, G. Gaul $\mathrm{Ar}-36, \mathrm{Ne}-20, \mathrm{C}-12,(\mathrm{C})$ $\mathrm{C}-13\left(\mathrm{CO}_{2}\right), \mathrm{N}-14\left[\left(\mathrm{NH}_{4}\right)_{2} \mathrm{SO}_{4}\right], \mathrm{O}-16\left(\mathrm{O}_{2}\right)$

236. Institut fur Kernphysik der Universitat Giessen Leihgesterner Weg 217

D-6300 Giessen, West Germany

$K$. Wienhard, U. Berg

$\mathrm{Ne}-22, \mathrm{~s}-34(\mathrm{~S})$ 


\section{WEST GERMANY}

237. Institute Fur Kern Physik

August-Euler-Str. 6

6 Frankfurt AM Main 90

West Germany

Mr. Schopper

Ar-38

238. Max-Planck-Institut fur Kernphysik Postfoch $10 \quad 39 \quad 80$

6900 Heidelberg

Germany

G. J. Wagner

$\mathrm{Ne}-20, \mathrm{Ne}-22, \mathrm{C}-13(\mathrm{C})$

239. Universitat zu Koln

Institut $f$. Kernphysik

Zulpicher st. 77

D-5000 Koln 41,

West Germany

w. Gruhle

Ar-36

INDIA

240. Electronics Corp. of India Ltd.

Industrial Development Area

Cherlapalli, Hyderabad-500 040

India

Y. G. Shankner

$\mathrm{He}-3, \mathrm{Ne}-20$

241. Govt. of India

Dept. of Atomic Energy

Directorate of Purchase \& Stores

Central Purchase Unit

Bombay 400 001, India

V. K. Chopra

242. Indian Institute of Science

Bangalore 560012

ri.a Madras (India)

$\mathrm{He}-3$, Ne-20

\section{ITALY}

243. Istituto Nazionale Fisica Nucleare 135100 Padora via Marzolo, 8

Italy

prof. G. Zannoni, C. Ceolin

244. Istituto Nazionale Fisica Nucleare Laboratori Nazionale di Legnaro

Via Romean. 4

35020 Legnaro (Padova) Italy

R. A. RiCCi
ISRAEL

245. Hebrew University of Jerusalem

Recah Inst. of Physics

Givat Ram, Jerusalem

91000 Israel

L. Tieberman

$\mathrm{C}-12(\mathrm{C})$

246. Seforad Applied Radiation, Ltd. Emek Hayarden

Israel

Y. Rapaport

247. Technion-Israel Institute of Technology

32000 Haifa, Israel

C. H. Lublin

248. Tel-Aviv University

Physics Dept.

Ramat-Aviv, Tel-Aviv, Israel

R. Rosenbaum, T. Katz

\section{JAPAN}

249. Daiwa Bank, Ltd.

Kinshicho Branch

Tokyo, Japan

T. Shimizu

250. ICHIBO Traders, Ltd.

No. 13-12 1-Chome, Ginza

Choo-Ku

Tokyo, Japan

'I'. Tanaka

251. Japan E.T.L. Company

4-9-6 Nakatsu Minamidori

Oyodo-Ku

Osaka, Japan

Sumi Yokoyama

252. Japan Radioisotope Association 28-45 Hon-Kómagoma 2-chome Bunkyo-ku, Tokyo, Japan

S. Yokoyama

$\mathrm{He}-3, \mathrm{Kr}-78, \mathrm{Kr}-80, \mathrm{Kr}-82$

$\mathrm{Kr}-83, \mathrm{Kr}-84, \mathrm{Kr}-86$

253. The Mitsubishi Bank Ltd.

Kamata Branch

Tokyo, Japan

F. Mochizuki

254. National Radiological Sciences

Institute

Tokyo, Japan

Sumi Yokoyama 
JAPAN

255. Okura \& Co., Ltd.

3-6 Ginza Nichome

Chuo-Ku, Tokyo, Japan

$\mathrm{K}$. Nakagawa

$\mathrm{He}-3$, Ne-20

256. Takachiho Trading Co., Ltd.

1-4-8 Hiroo Shibuyako

Tokyo, Japan

S. Matsumoto

$\mathrm{He}-3, \mathrm{Ar}-36, \mathrm{Kr}-84$

257. Tokyo University

Faculty of Science

Institute of Ind. Science

Inst. of Solid state Physics

Institute of Medical Science

Inst. for Nuclear studies

Tokyo, Japan

\section{NETHERLANDS}

258. Hoe Kloos

13058 Loosnl

Amsterdam, The Netherlands

T. Jacobs

259. Kernfysisch Versneller Instituut

Rijjksuniversiteit

Zernikelaan 25

Groningen, Netherlands

R. H. Siemssen

J. P. F. Mulder B. Koop

$\mathrm{He}-3, \mathrm{Ne}-20, \mathrm{Ne}-22, \mathrm{~N}-15\left(\mathrm{~N}_{2}\right)$

260. Twente University of Technology

Be stemming TN, Drienerlo

Enschede - The Netherlands

L. C. Vander Marel

261. Utrecht State University

Nuclear Physics Dept.

Sorbonnelaan 4, Utrecht

Netherlands

H. Colijnlaan

$0-18\left(\mathrm{H}_{2} \mathrm{O}\right)$

262. Voor Allegemene Natuurkundig

Laboratorium

der Riijksuniversitcit

Westersingel 34, Groningen

Netherlands

A. Hooper
NEW ZEALAND

263. Auckland, Univ of

Physics Dept.

Auckland, New Zealand

H. Naylor

264. Stores officer

(ED6024)

Education Dept.

Christchurch, New Zealand

E. M. Blaschke

\section{PHILIPPINES}

265. The International Rice Research Institute

P. O. Box 933

Manila, Philippines

P. Banzon

$\mathrm{N}-15\left(\mathrm{~N}_{2}\right)$

\section{SOUTH AFRICA}

266. Pretoria, University of Physics Dept. Pretoria, South Africa

o. Roetger

\section{SWEDEN}

267. $A B$ Atomenergi

Fack

61101 Nykoping;, Sweden

J. Lundgren

268. Chalmers University of Technology Physics Dept. S-402 20

Goteborg 5, Sweden

S. E. Arnell, E. Wallander

$\mathrm{Kr}-78, \mathrm{Kr}-80$, $\mathrm{Kr}-82$

269. Institute of Technology

Dept. of Solid state Physics

S-751 21 Uppsala, Sweden

o. Beckman 
SWITZERLAND

270. Bern, Universitat

Physikalisches Institut

Sidlerstrasse 5

3012 Bern, Switzerland

H. H. Loosli, O. Eugster

$\mathrm{He}-3, \mathrm{Ne}-22, \mathrm{Ar}-36, \mathrm{Ar}-37, \mathrm{Ar}-38$, $\mathrm{Kr}-78$

271. Cern

1211 Geneva 23

Switzerland

J. Lehmann, Mr. Blechschmidt

$\mathrm{He}-3, \mathrm{C}-13\left(\mathrm{CO}, \mathrm{CH}_{4}\right)$

272. Eidg. Technische Hochschule Laboratorium fur Kernphysik Honggerberg, 8049 Zurich, Switzerland

W. Gruebler
TAIWAN

273. Institute of Nuclear Energy

Research

P. O. Box 325

Lung-Tan, Taiwan

274. Precision Instrument Development Center

National Science Council

P. O. Box 2 No. 2 Post office

Kuang Fu Road Hsinchu, Taiwan 300

C. Chaug 


\section{State Index}

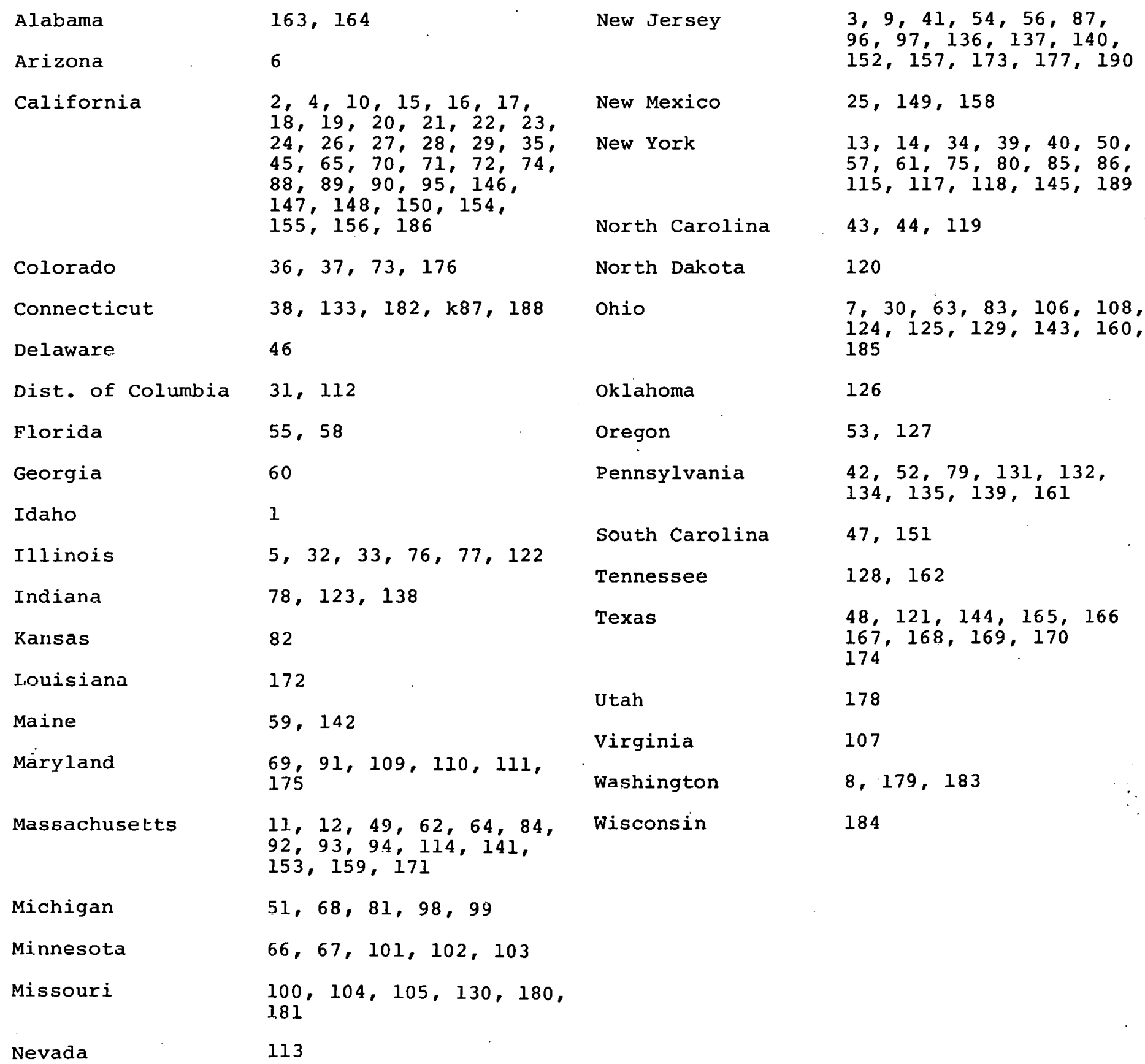


Isotope Index

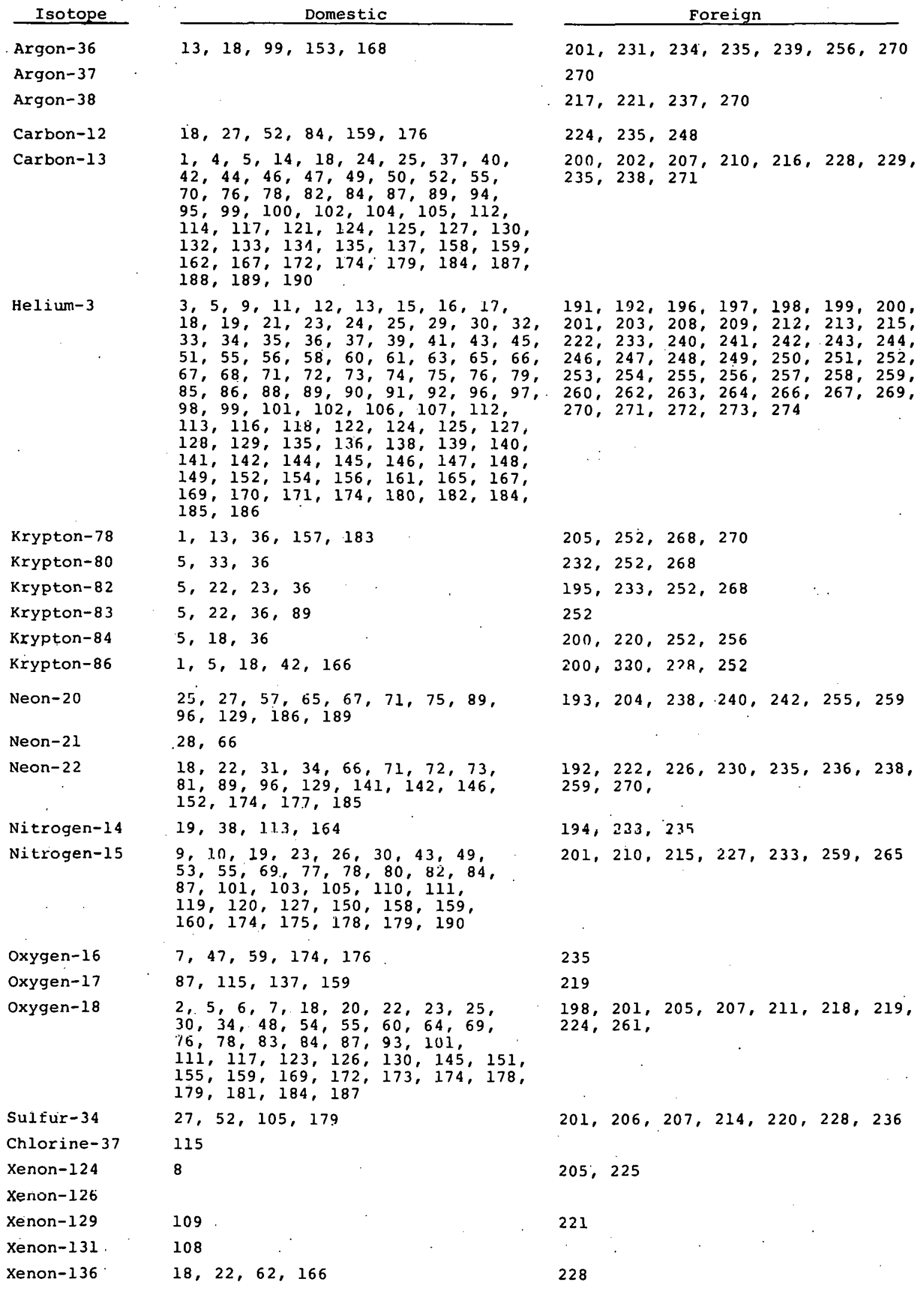


Stable Isotope Sales FY 1976 and FY 1976A

\begin{tabular}{|c|c|c|c|c|c|c|c|c|c|c|c|c|}
\hline \multirow[b]{2}{*}{ Isotope } & \multicolumn{3}{|c|}{ Domestic } & \multicolumn{3}{|c|}{ Foreign } & \multirow{2}{*}{$\begin{array}{l}\text { ERDA } \\
\text { Ship- } \\
\text { ments }\end{array}$} & \multirow{2}{*}{$\begin{array}{l}\text { and other } \\
\text { Liters } \\
\end{array}$} & \multirow{2}{*}{$\frac{\text { Federal }}{\text { Dollars }}$} & \multicolumn{3}{|c|}{ Total } \\
\hline & $\begin{array}{l}\text { Ship- } \\
\text { ments }\end{array}$ & Liters ${ }^{a}$ & Dollars & $\begin{array}{l}\text { Ship- } \\
\text { ments }\end{array}$ & Liters ${ }^{a}$ & Dollars & & & & $\begin{array}{l}\text { Ship- } \\
\text { ments }\end{array}$ & Liters ${ }^{a}$ & Dollars \\
\hline $\begin{array}{l}\text { Argon-36 } \\
\text { Argon-37 } \\
\text { Argon-38 }\end{array}$ & $\frac{2}{1}$ & $\begin{array}{l}.225 \\
.005\end{array}$ & $\begin{array}{c}1,027 \\
- \\
246\end{array}$ & $\begin{array}{l}7 \\
1 \\
3\end{array}$ & $\begin{array}{r}2.2 .70 \\
\text { Lot } \\
.115\end{array}$ & $\begin{array}{l}8,980 \\
1,238 \\
3,939\end{array}$ & $\begin{array}{l}4 \\
- \\
-\end{array}$ & $\begin{array}{c}5.145 \\
- \\
-\end{array}$ & $\begin{array}{c}13,411 \\
-\end{array}$ & $\begin{array}{r}13 \\
1 \\
4\end{array}$ & $\begin{array}{r}7.640 \\
\text { Lot } \\
.120\end{array}$ & $\begin{array}{r}23,418 \\
1,238 \\
4,185\end{array}$ \\
\hline $\begin{array}{l}\text { Carbon-12 } \\
\text { Carbon-13 } \\
\text { Carbon-13, LASL }\end{array}$ & $\begin{array}{r}5 \\
83\end{array}$ & $\begin{array}{r}91.990^{\mathrm{b}} \\
685.776^{\mathrm{b}}\end{array}$ & $\begin{array}{r}1,318 \\
55,564\end{array}$ & $\begin{array}{r}5 \\
27\end{array}$ & $\begin{array}{r}100.350^{\mathrm{b}} \\
1680.436^{\mathrm{b}}\end{array}$ & $\begin{array}{r}6,124 \\
114,383\end{array}$ & $\begin{array}{r}2 \\
20 \\
2\end{array}$ & $\begin{array}{r}7.080^{\mathrm{b}} \\
241.190^{\mathrm{b}} \\
1000 \mathrm{~g}\end{array}$ & $\begin{array}{r}200 \\
14,254\end{array}$ & $\begin{array}{r}12 \\
130 \\
2\end{array}$ & $\begin{array}{l}199.420^{b} \\
2,607.402^{b} \\
1,000 g\end{array}$ & $\begin{array}{r}7,642 \\
184,201\end{array}$ \\
\hline Helium-3 & 135 & 1855.070 & 255,858 & 81 & 949.000 & 156,438 & 33 & 3987.759 & 336,554 & 249 & $6,791.829$ & 748,850 \\
\hline $\begin{array}{l}\text { Krypton }-78 \\
\text { Krypton }-80 \\
\text { Krypton }-82 \\
\text { Krypton }-83 \\
\text { Krypton }-84 \\
\text { Krypton }-86\end{array}$ & $\begin{array}{l}1 \\
1 \\
2 \\
5 \\
1 \\
4\end{array}$ & $\begin{array}{l}.010 \\
.010 \\
.030 \\
.330 \\
.100 \\
.950\end{array}$ & $\begin{array}{r}441 \\
267 \\
383 \\
3,289 \\
201 \\
964\end{array}$ & $\begin{array}{l}6 \\
5 \\
5 \\
1 \\
4 \\
3\end{array}$ & $\begin{array}{l}.554 \\
.134 \\
.134 \\
.010 \\
.580 \\
.560\end{array}$ & $\begin{array}{r}6,487 \\
3,319 \\
1,410 \\
157 \\
1,130 \\
581\end{array}$ & $\begin{array}{l}5 \\
2 \\
6 \\
1 \\
3 \\
6\end{array}$ & $\begin{array}{r}1.240 \\
.085 \\
.910 \\
.100 \\
10.040 \\
30.040\end{array}$ & $\begin{array}{r}3,113 \\
1,315 \\
4,884 \\
703 \\
8,145 \\
11,922\end{array}$ & $\begin{array}{r}12 \\
8 \\
13 \\
7 \\
8 \\
13\end{array}$ & $\begin{array}{r}1.904 \\
.229 \\
1.074 \\
.440 \\
10.720 \\
31.550\end{array}$ & $\begin{array}{r}10,041 \\
4,901 \\
6,677 \\
4,149 \\
9,476 \\
13,467\end{array}$ \\
\hline $\begin{array}{l}\text { Neon-20 } \\
\text { Neon-21 } \\
\text { Neon-22 }\end{array}$ & $\begin{array}{r}11 \\
2 \\
12\end{array}$ & $\begin{array}{r}19.630 \\
.560 \\
18.500\end{array}$ & $\begin{array}{l}2,709 \\
7,735 \\
6,718\end{array}$ & $\frac{5}{6}$ & $\begin{array}{c}13.750 \\
- \\
12.510\end{array}$ & $\begin{array}{l}1,667 \\
3, \overline{123}\end{array}$ & $\frac{1}{3}$ & $\begin{array}{c}1.000 \\
48.000\end{array}$ & $-_{4,399}^{191}$ & $\begin{array}{r}17 \\
2 \\
21\end{array}$ & $\begin{array}{r}34.380 \\
.560 \\
79.010\end{array}$ & $\begin{array}{r}4,567 \\
7,735 \\
14,240\end{array}$ \\
\hline $\begin{array}{l}\text { Nitrogen-14 } \\
\text { Nitrogen-15 } \\
\text { Nitrogen-15, I.ASL }\end{array}$ & $\begin{array}{r}4 \\
55\end{array}$ & $\begin{array}{l}843.520^{c} \\
863.614^{b}\end{array}$ & $\begin{array}{l}92,911 \\
59,620\end{array}$ & $\begin{array}{r}3 \\
13\end{array}$ & $\begin{array}{r}13.000^{c} \\
257.075^{b}\end{array}$ & $\begin{array}{r}2,222 \\
18,782\end{array}$ & $\begin{array}{r}3 \\
14 \\
1\end{array}$ & $\begin{array}{l}438.940^{c} \\
766.246^{b} \\
200.9\end{array}$ & $\begin{array}{l}46,640 \\
35,139\end{array}$ & $\begin{array}{r}10 \\
82 \\
1\end{array}$ & $\begin{array}{l}1,29.5 .460^{c} \\
1,886.935 \\
200.9\end{array}$ & $\begin{array}{l}141,773 \\
113,541\end{array}$ \\
\hline $\begin{array}{l}\text { Oxygen-16 } \\
\text { Oxygen-17 } \\
\text { Oxygen-18 } \\
\text { Oxygen-18, LASL }\end{array}$ & $\begin{array}{r}1 \\
6 \\
52\end{array}$ & $\begin{array}{r}171.600^{\mathrm{b}} \\
.573^{\mathrm{b}} \\
128.416^{\mathrm{b}}\end{array}$ & $\begin{array}{r}533 \\
996 \\
17,169\end{array}$ & $\begin{array}{r}1 \\
1 \\
10\end{array}$ & $\begin{array}{r}42.900^{b} \\
.917^{b} \\
71.564^{b}\end{array}$ & $\begin{array}{r}236 \\
256 \\
7,942\end{array}$ & $\begin{array}{r}\frac{6}{-} \\
26 \\
1\end{array}$ & $\begin{array}{l}2941.800^{b} \\
783.160^{b} \\
100.9\end{array}$ & $\begin{array}{r}4,461 \\
- \\
57,538\end{array}$ & $\begin{array}{r}8 \\
7 \\
88 \\
1\end{array}$ & $\begin{array}{c}3,156.300^{\mathrm{b}} \\
1.490^{\mathrm{b}} \\
983.140^{\mathrm{b}} \\
100.9\end{array}$ & $\begin{array}{r}5,230 \\
1,252 \\
82,649\end{array}$ \\
\hline Sulfur-34 & 3 & 3.150 & 1,056 & 9 & 15.270 & 5,315 & 1 & .920 & 361 & 13 & 19.340 & 6,732 \\
\hline $\begin{array}{l}\text { Xenon-124 } \\
\text { Xenon-126 } \\
\text { Xenon-129 } \\
\text { Xenon-131 } \\
\text { Xenon-136 }\end{array}$ & $\begin{array}{l}- \\
- \\
- \\
1\end{array}$ & $\begin{array}{l}- \\
- \\
- \\
.015\end{array}$ & $\begin{array}{l}- \\
- \\
- \\
650\end{array}$ & $\begin{array}{l}4 \\
- \\
1 \\
-1 \\
1\end{array}$ & $\begin{array}{l}.780 \\
\overline{2} \\
.200 \\
\overline{0} \\
.020\end{array}$ & $\begin{array}{c}40,261 \\
- \\
681 \\
\overline{742}\end{array}$ & $\begin{array}{l}1 \\
\overrightarrow{1} \\
1 \\
9\end{array}$ & $\begin{array}{r}.010 \\
.100 \\
.050 \\
20.820\end{array}$ & $\begin{array}{r}151 \\
- \\
372 \\
448 \\
160,938\end{array}$ & $\begin{array}{r}5 \\
- \\
2 \\
1 \\
11\end{array}$ & $\begin{array}{c}.790 \\
- \\
.300 \\
.050 \\
20.856\end{array}$ & $\begin{array}{r}40,412 \\
- \\
1,053 \\
448 \\
162,330\end{array}$ \\
\hline Mixture & 22 & 230.422 & 32,472 & 6 & 125.575 & 19,761 & 5 & 38.600 & 3,880 & 33 & 394.597 & 56,113 \\
\hline Chlorine -37 & 1 & .555 & 276 & - & - & - & - & - & - & 1 & .555 & 276 \\
\hline Total & 410 & & 542,403 & 208 & & 405,174 & 157 & & 709,019 & 775 & & $.656,596$ \\
\hline
\end{tabular}

${ }^{a}$ Grams or kilograms $0 \equiv$ isotope as noted:

$$
\begin{aligned}
& { }^{\mathrm{b}} \text { Grams } \\
& { }^{\mathrm{K}} \text { Kilograms }
\end{aligned}
$$

dMaterial used at Los $\Lambda$ lamos Scientific Laboratory (LASL). 
USAGE OF CARBON,NITROGEN, OXYGEN, SULFUR, AND CHLORINE ISOTOPE FORMS

Enrichment and Form

Domestic

Foreign

ERDA

Total $\begin{array}{lllll}\text { Ship- Grams } & \text { Ship- Grams } & \text { Ship- Grams } & \text { Ship- } & \text { Grams } \\ \text { ments } & \text { Isotope ments } & \text { Isotope ments } & \text { Isotope ments } & \text { Isotope }\end{array}$ USAGE OF CARBON-12 FORMS FY 1976 AND FY 1976A
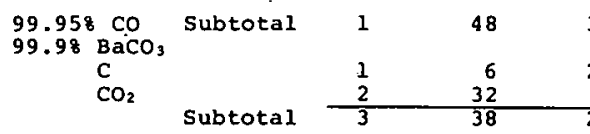

385 $\begin{array}{llll}2 & 15 & 1 & 6 \\ & 15 & 2 & 7\end{array}$ Total

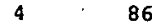

5

100

$3 \quad 13$

3

USAGE OF CARBON-13 FORMS FY 1976 AND FY 1976A

$998 \mathrm{C}$ (Includes LASL use)

$\mathrm{CO}_{2}$

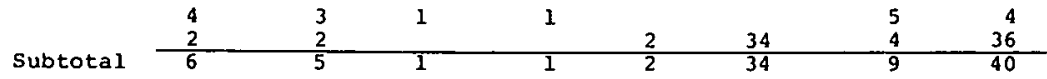

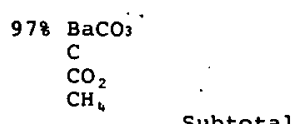

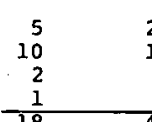

$908 \mathrm{BaCO}_{3}$
$\mathrm{C}$
$\mathrm{CO}_{2}$
$\mathrm{CO}$
$\mathrm{CH}_{4}$

Subtotal

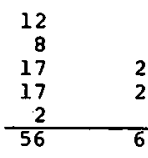

$208 \mathrm{CO}_{2}$

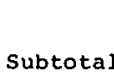

$\frac{3}{3}$

Total

$\begin{array}{llllll}83 & 691 & 27 & 1680 & 22 & 123\end{array}$

USAGE OF NITROGEN-14 FORMS FY 1976 AND 1976A

$99.998\left(\mathrm{NH}_{4}\right)_{2} \mathrm{SO}_{4}$

$\begin{array}{lllllll}4 & 844 & 3 & 13 & 3 & 439\end{array}$

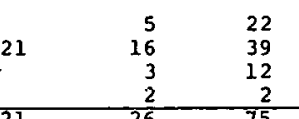

USACE OF NITROGEN-15 FORMS FY 1976 AND 1976A
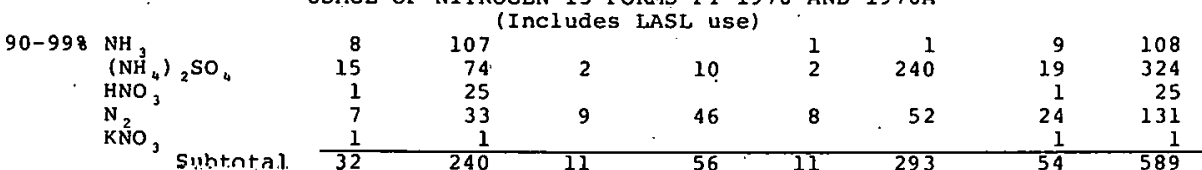

$658 \mathrm{KNO}_{3}$ Subtotal

40 \%
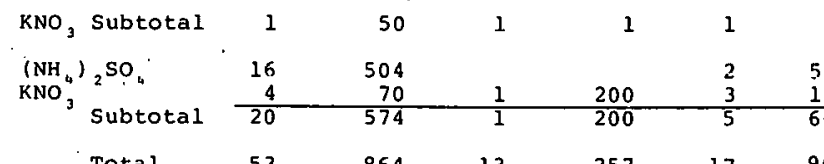

$6 \quad 3 \quad 37$

Total

USAGE OF OXYGEN-16 FORMS FY 1976 AND 1976A

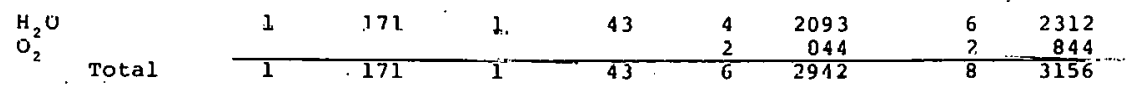

USAGE OF OXYGEN-17 FORMS FY 1976 AND FY 1976 A

$\begin{array}{lr}\mathrm{H}_{2} \mathrm{O} & \\ \mathrm{O}_{2} & \text { Total }\end{array}$

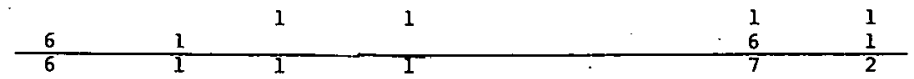

USAGE OF OXYGEN-18 FORMS FY 1976 AND FY 1976A

$$
\text { (Includes LASL use) }
$$

$\begin{array}{ll}\mathrm{H}_{2} & \\ \mathrm{O}_{2} & \text { Total }\end{array}$

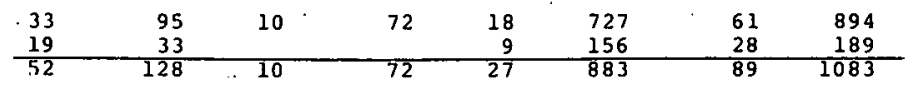

USAGE OF SUT.FUR-34 FORMS FY 1976 AIND $1976 \mathrm{~A}$

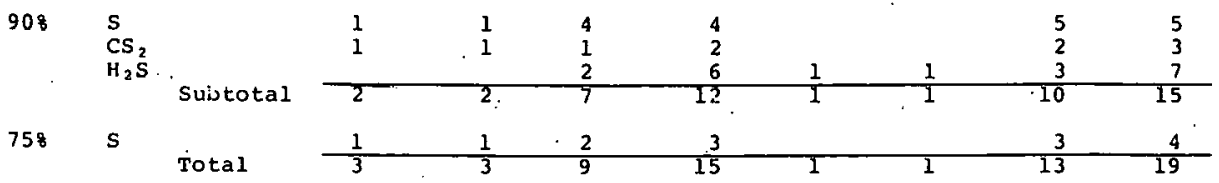

USAGD OF CHLORINE-37 FORMS FY, 1976 AND 1976A

$908 \quad \mathrm{NaCl}$ Total

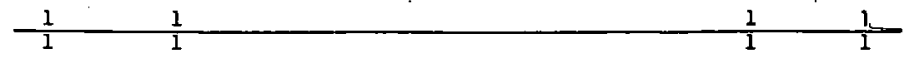




\title{
. Distribution
}

\author{
EXTERNAL \\ TID-4500, UC-23 (156) \\ G. Roggosa, ERDA/HQ (2) \\ W. Haubach, DPR, ERDA/HQ (6) \\ J. Maddox, DBER, ERDA/HQ (6) \\ R. Wood, DBER, ERDA/HQ (6) \\ D. Nowlin, ALO (3) \\ N. Matwiyoff, CNC-4, LASL (3) \\ J. A. Chacon, DAO (3) \\ R. K. Flitcraft, MRC
}

\section{INTERNAL}
V. L. Avona (40)
W. T. Cave
H. I. Charbeneau
C. W. Huntington
L. V. Jones
J. R. McClain
L. B. Stevens
R. E. Vallee
R. A. Schwind

Publications

Library (15) 